\title{
A novel spectrum allocation scheme in femtocell networks using improved graph theory
}

\author{
Feng Li* and Lili Yang ${ }^{2}$
}

College of Information Engineering, Zhejiang University of Technology, Hangzhou, 310023, China

\begin{abstract}
In this paper, we propose a novel spectrum allocation scheme by using graph coloring and taking QoS thresholds into account. The goal of our proposed method is to reduce interference among femtocells deployed densely and improve spectral efficiency after reaching user's QoS threshold. We design an interference-based graph model by detecting terminals' transmit power between femtocell stations and classifying the interference levels according to the coloring algorithm. Spectrum resource is allocated based on the QoS requirement of each user in a centralized way. Unlike other spectrum allocation method using graph theory, we take many significant factors into account in this paper in order to attain a more practical spectrum optimization solution. The simulation results show that when the scheme is deployed in femtocells with high density, it can effectively reduce the interference within same layer and improve system capacity along with spectrum efficiency.
\end{abstract}

Keywords: Femtocell, spectrum allocation, graph theory

Received on 04 November 2017, accepted on 07 December 2017, published on 13 December 2017

Copyright (C) 2017 Feng Li and Lili Yang, licensed to EAI. This is an open access article distributed under the terms of the Creative Commons Attribution licence (http://creativecommons.org/licenses/by/3.0/), which permits unlimited use, distribution and reproduction in any medium so long as the original work is properly cited.

doi: 10.4108/eai.13-12-2017.154701

"Corresponding author: Feng Li. Email: fenglzj@zjut.edu.cn

\section{Introduction}

Research shows that in recent years, the worldwide mobile data traffic has surged 13 times [1]. Other outcomes present that about two-thirds of mobile phone communications and over $70 \%$ of data traffic occur indoors [2]. Therefore, the indoor coverage quality of wireless signal becomes critical for user's experience. However, the macro base station usually cannot satisfy users' requirements for indoor traffic. Besides, mobile operators need to meet the requirement of customer service quality at a lower cost. Femtocell, as a low-power base station, becomes an ideal solution to enhance user's service experience indoor. In general, users can install the femtocell station in their rooms according to various needs. It is a mobile base station with high service quality, low power consumption, high stability and small coverage. It can provide consumers with good network services and transmission quality in given region. On the other hand, the application of femtocells also requires to solve many challenges [3]. As the distance between each household is always very close, the interference between them becomes very heavy especially when the density of the femtocells increases. Hence, it is worth to study how to mitigate the interference between femtocells within the same layer while ensuring the QoS requirements of all the users involved.

The same-layer interference between adjacent femtocells can be restrained by rational spectrum allocation scheme. In [4], the author adopted a spectrum allocation scheme which clusters the base stations. The control center of the femtocell networks uses the weighted interference map to allocate the base stations to the user groups and then authorizes the sub-bands to the base stations in dynamic way. The key point of this method is SINR-based clustering. In [5], the control center of the femtocell networks used the vertex coloring algorithm of graph theory, allocating sub-bands to each base station, and its sub-bands were not used by neighboring base stations. However, neither [4] nor [5] take into account the load of each base station. The spectrum allocation scheme proposed in [6] had deficiencies in sub-band 
allocation, although it can overcome the above disadvantages.

In this paper, we propose a spectrum resource allocation scheme that combines graph theory and QoS. The scheme is based on the coloring algorithm in the graph theory to realize the grouping of the base station, and then allocates spectrum re-sources according to the user's QoS requirements. Specifically, each base station classifies the base stations with large interference as interference neighbors and reports them to the center base station. And the center base station establishes the interference graph according to these interference relations, uses a specific algorithm to color. Base stations with the same color are grouped in the same group and reuse the same frequency. Different groups of base stations use mutually orthogonal sub-bands, thus greatly reducing the interference in the same layer and also improving the spectrum utilization and the femtocell network capacity.

The remainder of this article is organized as follows. In Section II, we introduce the system model. Section III describes the spectrum resource allocation scheme that combines graph theory and QoS. In Section IV, the simulation results and analyses are introduced. Finally, we conclude this paper in Section V.

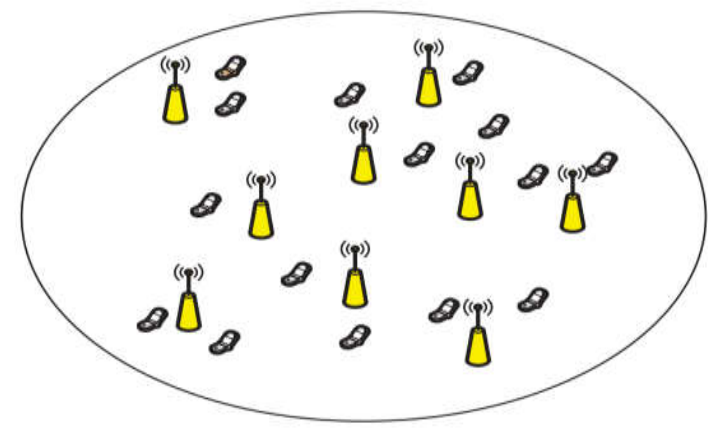

Fig. 1. System model of femtocell networks

\section{System Model}

In this paper, we consider downlink transmission based on orthogonal frequency division multiple access (OFDM), as shown in Figure 1. Femtocell and macrocell use different spectrum resources so that the cross-layer interference in the femtocell network can be ignored. The femtocell system controller (FSC) is a central controller that controls the configuration and status of femtocell base stations (FBSs) in the network. Throughout the femtocell network, the FSC controls numbers of FBSs, $N=\{1,2, \ldots, N\}$, which are randomly placed in each building and have a maximum coverage radius of $R c$ .Each activated FBS $i(i \in N)$ service has a set of FUEs within its coverage, $K i=\{1,2, \ldots, k i\}$, all of which are random locations. In the femtocell networks, the user number can be expressed as

$$
K=\sum_{i=1}^{i=N} K_{i}
$$

Femtocell base stations are densely deployed, so there e xists a heavy interference between them. We must identif y each FBS interference neighbors. For example, for user $\mathrm{FUE} k$ in $\mathrm{FBS} i \bar{l}$, if the interference of FBS $i$ to user FUE $k$ exceeds the threshold $\gamma_{t h}$, the FBS $j$ and FBS $i$ are mutu ally interference neighbors.

$$
\frac{\sum_{k=1}^{k=k_{i}} P_{i} g_{i, k}}{\sum_{k=1}^{k=k_{i}} P_{j} g_{j, k}}<\gamma_{t h} \quad i \neq j \in N
$$

where $P_{i}$ is the transmit power of FBS $i, g_{i, k}$ is the channel gain between FBS $i$ and FUE $k$. Then, the signal-tointerference and noise ratio (SINR) of user FUE $k$ in FBS $i$ can be expressed as

$$
\gamma_{i k}=\frac{P_{i} g_{i, k}}{\sum_{i \neq j} P_{j} g_{j, k}+\sigma_{k}^{2}}
$$

where $\sigma_{k}^{2}$ is white noise power. We assume that the total spectral bandwidth of the system in the femtocell network is $B$. FSC divides $B$ into a group of same sub-bands of ba ndwidth, $S=[1,2, \ldots, 5]$, and their bandwidth is $B_{S}$. Then , given a specific $\gamma_{i k}$, the spectral efficiency (in bps / Hz) of FBS $i$ to FUE $k$ in the unit bandwidth can be given as

$$
R_{i k}=\left\{\begin{array}{ccl}
0 & \text { for } & \gamma_{i k}<\gamma_{\min } \\
\alpha \log _{2}\left(1+\gamma_{i k}\right) & \text { for } & \gamma_{\min }<\gamma_{i k}<\gamma_{\max } \\
R_{\max } & \text { for } & \gamma_{\max }<\gamma_{i k}
\end{array}\right.
$$

where $\gamma_{\min }$ is minimal SINR. We set $\gamma_{\text {min }}=-1 d B, \gamma_{\max }=19.5 d B$ as maximal SINR. $\alpha=0.6$ denotes the attenuation factor.

In order to meet the QoS needs of each user, the base station serving the user must provide sufficient bandwidth. In this article, FBS $i$ must provide enough subbands for the FUE $k$ it serves. The demand sub-band for each FBS $i$ is given by the following formula

$$
\begin{gathered}
r_{i k}=R_{i k} B_{S} \\
B_{i k}=\frac{Q_{r}}{r_{i k}} \\
B_{i}=\left\lceil\sum_{k=1}^{k=k_{i}} B_{i k}\right\rceil, \forall i \in N
\end{gathered}
$$

where $r_{i k}$ is the reachable rate of FBS $i$ to FUE $k$ in a subband under a specific $\gamma_{i k} . Q_{r}$ is the Qos requirement of user FUE $k, B_{i k}$ is the required bandwidth of FUE $k$ for 
each user served by FBS $\bar{i} . \mathbb{B}_{\tilde{i}}$ denotes the desired bandwidth for FBS $i$.

\section{Spectrum Resource Allocation Scheme}

In this section, we propose a spectrum allocation scheme (CGQR) that combines graph theory and QoS. The scheme is composed of three steps: Interference graph formation and coloring; base station grouping based on the coloring result; the allocation of spectrum resources.

- Step 1: Interference graph formation and coloring

In this step, we first obtain the interference graph of the base station according to the formula (2), establish the adjacency matrix, and utilize the graph theory algorithm for the vertex shading.

1) When the FSC detects FBS activation, the active base station reports its neighbor list to the FSC. FSC constructs the interference graph $G=(V, E), \quad V$ represents the set of vertices of FBS, and $E$ represents the set of edges of the interference between two FBSs. We represent the relationship between base stations as a binary matrix, i.e. the adjacency matrix $W$. This matrix can be expressed as $W-\left[w_{i j}\right]_{N r W}$.

$$
\omega_{i j}= \begin{cases}1 & F B S i \text { and FBS } j \text { are neighbors } \\ 0 & \text { Otherwise }\end{cases}
$$

where $\omega_{i j}=1$ indicates that the base station FBS $i$ and

FBS $j$ are mutually interference neighbors. Otherwise, interference can be tolerated.

2) After the interference graph is established, we will use the DSATUR algorithm [7] to color the vertices in the graph. The algorithm has two important

parameters: vertex saturation $\mathcal{C}$ and vertex degree $\theta$. $\theta$ represents the number of adjacent vertices of different colors, and $\theta$ represents the number of adjacent vertices. Note that the colors of adjacent vertices are different.

- Step 2: Group base stations based on the coloring result

In this step, we group the base station based on the coloring result in the previous step. Base stations with the same color are grouped in the same group, otherwise, grouped in different groups. After grouping, there are k groups, $\quad G=[1,2, \ldots, k]$.

- Step 3: Allocation of spectrum resources

After all the base station have been grouped, find the desired bandwidth for each group:

$$
B_{k}=\sum_{i \in G_{k}} B_{i}
$$

where $G_{k}$ is the set of base stations in the $k$ th group. Since the number of users in each base station may be different and the QoS of each user may be different, the average required bandwidth for each group is

$$
B_{a k}=\frac{B_{k}}{n_{k}}
$$

where $n_{k}$ is the number of FBS in the $k t h$ group. Then, the actual bandwidth allocated to each team is

$$
B_{c a k}=S^{*} \frac{B_{a k}}{\sum_{k \in G} B_{a k}} * B_{S}
$$

Although the bandwidth allocated to each base station may be less than its required bandwidth, it minimizes interference and meets its QoS needs, as well as increasing spectrum efficiency. Of course, the above spectrum resource allocation scheme needs to satisfy the condition of $|C|<s$.

\section{Numerical Results}

In this section, we simulate the scheme proposed in the third part and analyze the numerical results. Regarding the simulation environment, we use the downlink transmission in the femtocell network. The femtocell network model used in this paper is a $5 \times 5$ grid. Use a non-interference single story apartment building, this floor a total of 25 apartments, an area of $10 \mathrm{~m} \times 10 \mathrm{~m}$. One FBS is deployed randomly in each apartment. FSC can control all FBS in this layer and coordinate resource sharing between FBS. The activation probability of FBS is $P_{a}$. For example when $P_{a}=0.2,5$ of $25 \mathrm{FBS}$ are activated and their positions are randomly distributed. We assume that there is only the same layer of interference between these FBS, so the impact from the macro base station can be ignored. The SINR threshold $\gamma_{\text {th }}$ we use to construct the interference graph is set to $4,4 d B$.The simulation parameters are given in Table 1.

\section{Table 1. Simulation parameters}

\begin{tabular}{cc}
\hline System Bandwidth & $10 \mathrm{MHZ}$ \\
\hline $\begin{array}{c}\text { Carrier frequency } \\
\text { Number of available sub- } \\
\text { bands }\end{array}$ & $2 \mathrm{GHz}$ \\
\hline
\end{tabular}




\begin{tabular}{cc}
\hline Sub-band bandwidth & $180 \mathrm{KHz}$ \\
FBS coverage radius & $20 \mathrm{~m}$ \\
Coverage of the building & $50 \mathrm{~m} * 50 \mathrm{~m}$ \\
Minimum distance & \\
between FBSs & $2 \mathrm{~m}$ \\
FBS transmission power & $20 \mathrm{dBm}$ \\
Path loss & $127+30 \log 10(\mathrm{r} / 1000) \mathrm{r} / \mathrm{m}$ \\
Noise power density & $-174 \mathrm{~dB} \mathrm{~m} / \mathrm{Hz}$ \\
Minimum distance \\
between FBS and FUE
\end{tabular}

Compare our proposed spectrum resource allocation plan (CGQR) with two scenarios:

- ASR: All FBS reuse all sub-bands, all spectrum reuse;

- SEDR: After FBS is grouped, the number of subbands allocated to each group is the ratio of the total number of sub-bands to the number of groups, that is, spectrum evenly distributed reuse;

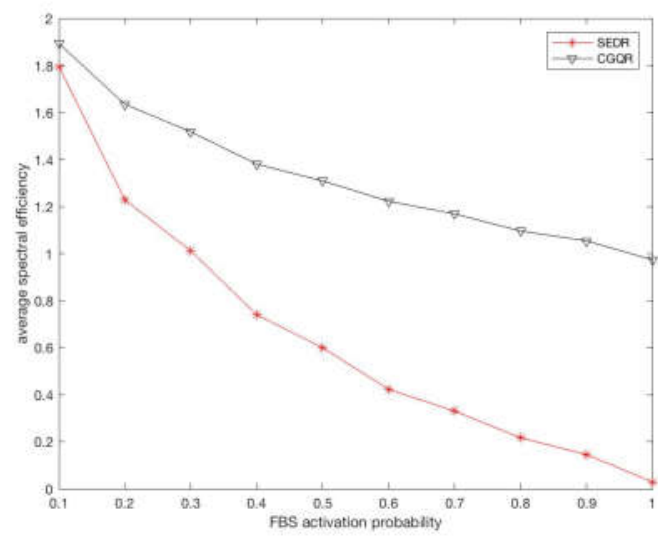

Figure 2. Average spectral efficiency with activation probability

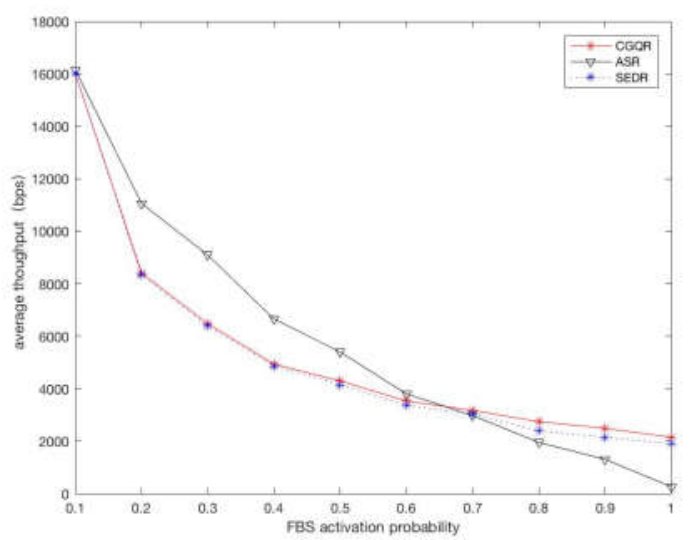

Figure 3. Average throughput with activation probability
Average spectral efficiency: We compare the spectral efficiency between the three schemes. Since both CGQR and SEDR use the coloring algorithm in section 3, the grouping results are the same. Therefore, only the average spectral efficiency between CGQR and ASR is compared. We assume that all FBSs in the femtocell network use closed access mode and only serve FUE within their apartment. For the sake of simplicity, let's assume that the number of fuels in each apartment is 1

and the QoS needs are all 384kbps. As shown in Figure 2, the spectral efficiency of the three schemes shows a decreasing trend due to the increase of femtocell base station density, but the CGQR curve will drop slowly,

especially when $P_{\mathbb{R}}$ increases. Femtocell base station density increases, the interference between them also increases, our scheme has obvious advantages in antiinterference aspect. Therefore, our proposed scheme can significantly improve the average spectral efficiency of the femtocell network when the femtocell network density is high.

Average Throughput: We still assume that the number of FUEs in each apartment is 1, which all require

384kbps for QoS. As shown in Fig.3, when $P_{Z}$ is small, since the femtocell base station has a small density, and the ASR can use all the sub-bands, the interference at the same layer is light, so the throughput is better than the

solution we propose. As $P_{Q}$ increases, the femtocell base station density increases, and the average throughput of CGQR and SEDR begins to be greater than the ASR. Because our solution is based on QoS allocated on demand, and SEDR is evenly distributed, do not have the flexibility. So our scheme is slightly better than SEDR in the case of strong interference. Therefore, our proposed scheme is superior to the other two schemes when the femtocell base station density is high.

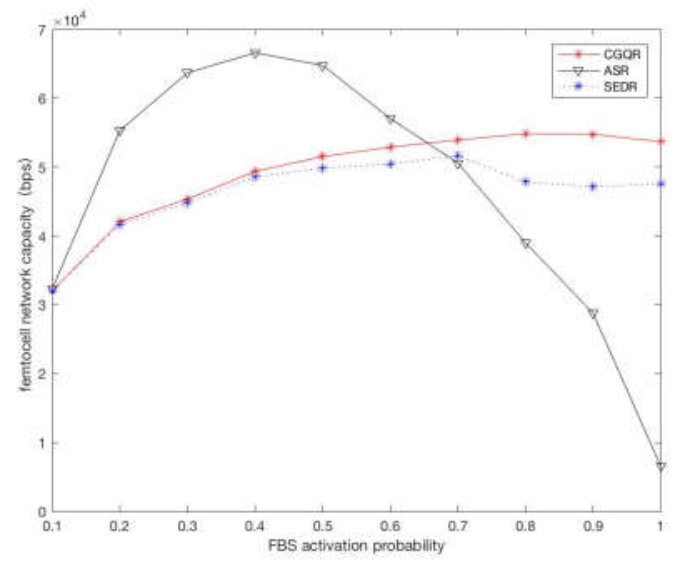

Figure 4. Femtocell network capacity with activation probability 
Femtocell Network Capacity: We continue to set the number of FUEs in each apartment to 1, which all require $384 \mathrm{kbps}$ for QoS. As shown in Fig. 4, as the number of activated base stations in the femtocell network increases, the network capacity in the ASR scheme becomes larger, reaching a peak when $P_{\Omega}=0.4$. After that, as the femtocell base station density increases, and the interference in the same layer becomes larger, the capacity of the network begins to drop drastically. The network capacity of CGQR and SEDR has been on a steady upward trend. In particular, the solution proposed by us has almost no fluctuation in the upward trend of network capacity, indicating that CGQR has obvious advantages over the other two schemes in terms of anti-interference. When the base station density becomes larger and the number of users increases, the total capacity of the proposed scheme in the network is obviously better than the other two schemes.

\section{Conclusions}

In this paper, we propose a spectrum resource allocation scheme which adopts graph theory and takes QoS requirement into account. The base stations in femtocell networks are first analogized to the vertices in the graph theory and then colored. The base stations with the same color are grouped, and then sub-bands are allocated to each group of base stations according to the QoS requirement of each FUE. The simulation results show that when the system load becomes larger, the antiinterference ability, system throughput and spectrum efficiency of our proposed scheme are better than the other traditional methods. Besides, there are still many shortcomings in our algorithm which need to be solved in future work.

\section{References}

[1] Cisco visual networking index: Global mobile data traffic forecast update, in Whitepaper, Feb. 2013.

[2] X Zhou, S L Feng, Y Ding, et al. Game-theoretical frequency reuse algorithm in Femtocell network. Journal on Communications, 2015, 36(2): 137-143.

[3] J. Andrews, Seven ways that hetnets are a cellular paradigm shift, IEEE Communications Magazine, vol. 51, no. 3, pp. 136-144, 2013.

[4] S. Uygungelen, G. Auer, and Z. Bharucha, Graph-based dynamic frequency reuse in femtocell networks, in Proc. 2011 IEEE VTC-Spring, pp. 1-6.

[5] S. Kim, and I. Cho, Graph-Based Dynamic Channel Assignment Scheme for Femtocell Networks, IEEE Communication Letters, vol. 17, no. 9, pp. 1718-1721, 2013.

[6] P. Demestichas, A. Georgakopoulos, D. Karvounas, et al, $5 \mathrm{G}$ on the horizon: Key challenges for the radio-access network, IEEE Vehicular Technology Magazine, vol. 8, no. 3, pp. 47-53, 2013.

[7] D. Brelaz, New methods to color the vertices of a graph, Communications of the ACM, vol. 22, no. 4, pp. 251-256, 1979. 